\title{
Difluoroboron $\beta$-diketonate polylactic acid oxygen nanosensors for intracellular neuronal imaging
}

\author{
Meng Zhuang ${ }^{1}$, Suchitra Joshi ${ }^{2}$, Huayu Sun ${ }^{2}$, Tamal Batabyal ${ }^{2}$, Cassandra L. Fraser ${ }^{1 \bowtie}$ \& \\ Jaideep Kapur ${ }^{2,3,4 \bowtie}$
}

Critical for metabolism, oxygen plays an essential role in maintaining the structure and function of neurons. Oxygen sensing is important in common neurological disorders such as strokes, seizures, or neonatal hypoxic-ischemic injuries, which result from an imbalance between metabolic demand and oxygen supply. Phosphorescence quenching by oxygen provides a non-invasive optical method to measure oxygen levels within cells and tissues. Difluoroboron $\beta$-diketonates are a family of luminophores with high quantum yields and tunable fluorescence and phosphorescence when embedded in certain rigid matrices such as poly (lactic acid) (PLA). Boron nanoparticles (BNPs) can be fabricated from dye-PLA materials for oxygen mapping in a variety of biological milieu. These dualemissive nanoparticles have oxygen-insensitive fluorescence, oxygen-sensitive phosphorescence, and rigid matrix all in one, enabling real-time ratiometric oxygen sensing at micron-level spatial and millisecond-level temporal resolution. In this study, BNPs are applied in mouse brain slices to investigate oxygen distributions and neuronal activity. The optical properties and physical stability of BNPs in a biologically relevant buffer were stable. Primary neuronal cultures were labeled by BNPs and the mitochondria membrane probe MitoTracker Red FM. BNPs were taken up by neuronal cell bodies, at dendrites, and at synapses, and the localization of BNPs was consistent with that of MitoTracker Red FM. The brain slices were stained with the BNPs, and the BNPs did not significantly affect the electrophysiological properties of neurons. Oxygen maps were generated in living brain slices where oxygen is found to be mostly consumed by mitochondria near synapses. Finally, the BNPs exhibited excellent response when the conditions varied from normoxic to hypoxic and when the neuronal activity was increased by increasing $\mathrm{K}^{+}$concentration. This work demonstrates the capability of BNPs as a non-invasive tool in oxygen sensing and could provide fundamental insight into neuronal mechanisms and excitability research.

Molecular oxygen $\left(\mathrm{O}_{2}\right)$ plays an essential role in living systems ${ }^{1,2}$. In eukaryotic cells, oxygen is required as a terminal electron acceptor in the aerobic metabolic process, known as oxidative phosphorylation ${ }^{3}$. This reaction takes place in mitochondria, during which the electrons transfer from an electron donor to an electron acceptor or oxygen, and energy released from electron flow is used to transform adenosine diphosphate (ADP) to adenosine triphosphate (ATP). The ATP generated during phosphorylation drives many cellular activities and processes and maintains biochemical function.

Molecular $\mathrm{O}_{2}$ is essential in the structural and functional integrity of the brain. Though the brain is only $2 \%$ of the body's mass, it consumes nearly $20 \%$ of $\mathrm{O}_{2}$ in the resting body ${ }^{4}$. Neuronal computation is energetically expensive, which requires an adequate supply of ATP or $\mathrm{O}_{2}$ for activities ranging from maintaining electrochemical gradients to releasing and recycling synaptic vesicles ${ }^{5,6}$. Hypoxia-ischemia is a major cause of brain injury in humans, including conditions such as hypoxic-ischemic encephalopathy ${ }^{7}$, stroke ${ }^{8,9}$, seizures ${ }^{8,10,11}$, periventricular leukomalacia ${ }^{12}$, and even Alzheimer's disease ${ }^{13,14}$. Therefore, it is important to understand neuronal oxygen levels in various neurological disorders.

Oxygen sensing with good spatial and temporal resolution can offer new fundamental insight into brain function. Current methods to assess $\mathrm{O}_{2}$ levels in biomedicine include polarographic oxygen electrodes (also known as Clark electrodes), radioisotope techniques (positron emission tomography and single-photon emission

\footnotetext{
${ }^{1}$ Department of Chemistry, University of Virginia, Charlottesville, VA 22904, USA. ${ }^{2}$ Department of Neurology, University of Virginia, Charlottesville, VA 22903, USA. ${ }^{3}$ Department of Neuroscience, University of Virginia, Charlottesville, VA 22903, USA. ${ }^{4}$ UVA Brain Institute, University of Virginia, Charlottesville, VA 22903, USA. ${ }^{\boxplus e m a i l: ~}$ cf4n@virginia.edu; jk8t@virginia.edu
} 
computed tomography), magnetic resonance techniques (e.g., fMRI), and optical methods based on hemoglobin $\mathrm{O}_{2}$ saturation and phosphorescence quenching ${ }^{15}$. Some of these methods have significant limitations of equipment cost, invasiveness, or low spatial resolution. For example, polarographic electrodes are invasive and limited to single-point measurements; radioisotope techniques only show regions of hypoxia. Also, hemoglobinbased detection is indirect and only provides an estimation of $\mathrm{O}_{2}$ levels in the blood; and fMRI assessments are restricted to long time scales.

Oxygen nanosensors based on phosphorescence quenching can address some of these limitations of spatial and temporal resolution with improved micron-level spatial and sub-seconds temporal resolution ${ }^{16-20}$. Efforts have been made during the past decades in applying phosphorescence probes as oxygen sensors in neuronal tissue $^{21-23}$. For example, Ingram et al. developed platinum (II) octaethylporphine ketone (PtOEPK) and nanocrystals quantum dot (NQD) blends to measure the interstitial oxygen concentration of active brain slices through fluorescence resonance energy transfer (FRET) ${ }^{24}$. The Papkovsky group designed cell-permeable probes based on a phosphorescent Pt(II)-tetrakis(pentafluorophenyl)porphyrin (PtPFPP) dye and a fluorescent poly $(9,9$ diheptylfluorene-alt-9,9-di-p-tolyl-9H-fluorene standard ${ }^{25}$. These methods rely on blending a phosphorescence probe, a fluorescence standard, and a polymer matrix altogether, rendering the synthetic and preparation process somewhat complex. In addition, currently, there are few reports measuring intraneuronal oxygen levels.

Difluoronboron $\beta$-diketonates are a class of compounds with excellent luminescence properties, including large extinction coefficients, high quantum yields, and two-photon absorption. Their emission is easily tunable by the chemical structure to span across the visible range, making them useful as bioimaging reagents. When a boron dye is confined in a rigid matrix such as poly (lactic acid) (PLA), the fluorescence is retained, and at room temperature, the phosphorescence is greatly enhanced ${ }^{26}$. This excellent feature offers an opportunity for boron dye polymers to serve as ratiometric oxygen sensors, where the oxygen-insensitive fluorescence is the internal standard for self-calibration, and the quenchable oxygen phosphorescence senses response on local oxygen level changes. With the presence of biocompatible and biodegradable PLA, the boron dye polymers can be fabricated in multiple ways for biological applications, including nanoparticles, thin-film, and nanofibers ${ }^{27}$. As the fluorescence standard, the phosphorescence sensor and the matrix are all combined in a single macromolecular material, boron dye polymers show unique advantages over many other phosphorescent probes. First, the dye preparation and fabrication process are cost-effective and facile within a few steps. Second, in contrast to the boron system, for ratiometric oxygen sensing, where the standard and the sensor are two separate compounds, it can be challenging to have the same photobleaching rate for both dyes in quantitative analysis. Also, both the boron dye and the polymer can be easily adapted for commonly used imaging setups and multiple biological purposes. In this work, the oxygen sensing capability of iodine substituted difluoroboron $\beta$-dibenzoylmethane PLA $\left(\mathrm{BF}_{2} \mathrm{dbm}(\mathrm{I}) \mathrm{PLA}\right)$ nanoparticles was employed to study brain activity. In particular, the brain slices were labeled by the cell-penetrating nanoparticles, and ratiometric oxygen maps were generated. It was found that the neuronal cell bodies consume less oxygen than dendrites and synapses, where mitochondria are more concentrated.

\section{Results and discussions}

Nanoparticles. The boron dye polymers were synthesized as previously described ${ }^{26}$. In general, the boron dye with a primary alcohol initiator group was dissolved in melted lactide. The polymers were grown in the presence of tin catalyst via ring-opening polymerization. The crude polymers were purified by dissolving in a minimal amount of $\mathrm{CH}_{2} \mathrm{Cl}_{2}$, followed by addition into cold methanol to remove residual small organic dyes and lactide. The polymers were characterized by NMR and GPC. The nanoparticles were fabricated by a method known as nanoprecipitation, which involves interfacial deposition due to displacement of solvent (i.e., DMF) with non-solvent (i.e., DI water). In this work, two types of nanoparticles were fabricated and characterized as illustrated in Fig. 1. Blue nanoparticles $\mathrm{BF}_{2} \mathrm{dbmPLA}$ (blue NPs) were chosen for labeling primary neuronal cells because their high quantum yield and bright fluorescence make them easily detected and ideal for cell tracking ${ }^{27}$. Oxygen sensing nanoparticles $\mathrm{BF}_{2} \mathrm{dbm}(\mathrm{I}) \mathrm{PLA}\left(\mathrm{O}_{2} \mathrm{NPs}\right)$ were used to generate ratiometric oxygenation maps where the fluorescence $\left(\lambda_{\mathrm{F}}=450 \mathrm{~nm}\right)$ acts as an internal standard and the oxygen-sensitive phosphorescence $\left(\lambda_{\mathrm{P}}=535 \mathrm{~nm}\right)$ is the sensor. Nanoparticle characteristics have been extensively studied in previous reports, as listed in Table 1. The maximum UV excitation wavelengths for both nanoparticles (blue NPs: $\lambda_{\text {ex }}=396 \mathrm{~nm} ; \mathrm{O}_{2}$ NPs: $\lambda_{\text {ex }}=405 \mathrm{~nm}$ ) are compatible with $405 \mathrm{~nm}$ laser excitation.

The physical properties, including radius and zeta potential, were measured by DLS and ZetaSizer. It is believed that size and surface charge are critical factors affecting the interaction between nanomaterials and biological components, consequently the efficiency of localization into cells and tissue. As listed in Table 1, the hydrodynamic radius for blue NPs was $52 \mathrm{~nm}$ with a polydispersity of $15 \%$, and for $\mathrm{O}_{2} \mathrm{NPs}$, it was $71 \mathrm{~nm}$ with a polydispersity of $24 \%$, both of which are typical values for the family of $\mathrm{BF}_{2}$ bdkPLA and are consistent with previous results ${ }^{28,29}$. The data suggested the polymers successfully self-assemble into nanoparticles. In addition, nanoparticles with radii ranging from 20 to $200 \mathrm{~nm}$ can be readily internalized via endocytosis; they are large enough to avoid renal clearance and small enough for clearance from the spleen ${ }^{30}$. Based on zeta potential, blue NPs and $\mathrm{O}_{2}$ NPs were negatively charged, in accordance with commonly developed PLA nanoparticles. An early report from Dante et al. revealed that NPs with negative charge rapidly localize on neuronal membranes, while those with positive or neutral charge showed no or slow interaction with neurons ${ }^{31}$. With the size and zeta potential suitable for endocytosis, we hypothesized that the boron dye polymer NPs would be internalized by neurons and respond to intracellular oxygen variations.

Cellular uptake. To confirm the intracellular uptake, the stability (i.e., no aggregation) of NPs was firstly evaluated in a neuronal medium at $37^{\circ} \mathrm{C}$ over time as nanoparticles fabricated from pure hydrophobic polyesters are prone to precipitate in an aqueous solution. The NPs were diluted with neuronal medium (included in 
A<smiles>CC(O)C(=O)OCCOc1ccc(C(=CC(=O)P(F)F)OCCCO)cc1</smiles>

$\mathrm{BF}_{2} \mathrm{dbmPLA}$<smiles>O=C(O)C(=O)OCCOc1ccc(C2=CC(c3ccc(I)cc3)=[O+][B-](F)(F)O2)cc1</smiles>

$\mathrm{BF}_{2} \mathrm{dbm}(\mathrm{I}) \mathrm{PLA}$

B

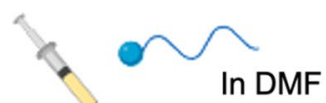
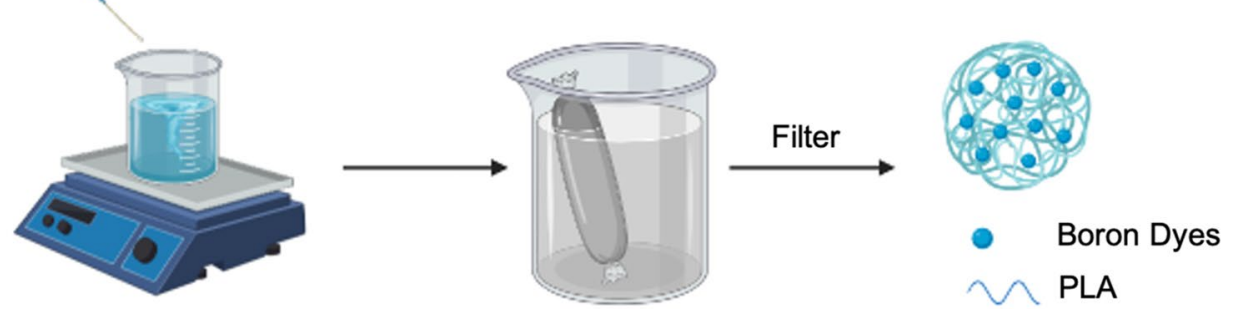

Figure 1. Nanoparticle composition and fabrication. (A) Chemical structures of blue-emitting $\mathrm{BF}_{2} \mathrm{dbmPLA}$ and dual-emissive, oxygen sensing $\mathrm{BF}_{2} \mathrm{dbm}(\mathrm{I}) \mathrm{PLA}$. (B) Nanoparticles are fabricated by the nanoprecipitation method.

\begin{tabular}{|c|c|c|c|c|c|c|c|c|}
\hline \multirow[b]{2}{*}{ Nanoparticles } & \multicolumn{5}{|c|}{ Optical properties } & \multicolumn{3}{|c|}{ Physical properties } \\
\hline & $\lambda_{\text {ex }}{ }^{\mathrm{a}}(\mathrm{nm})$ & $\lambda_{\mathrm{F}}^{\mathrm{b}}(\mathrm{nm})$ & $\lambda_{\mathrm{P}}{ }^{\mathrm{c}}(\mathbf{n m})$ & $\tau_{\mathrm{F}}^{\mathrm{d}}(\mathbf{n s})$ & $\tau_{\mathrm{P}}{ }^{\mathrm{e}}(\mathrm{ms})$ & $\mathbf{R}_{h}{ }^{\mathrm{f}}(\mathbf{n m})$ & $\mathbf{P D}^{\mathrm{f}}(\%)$ & $\zeta^{\mathrm{g}}(\mathrm{mV})$ \\
\hline $\mathrm{BF}_{2} \mathrm{dbmPLA}$ & 396 & 439 & 509 & 3.84 & 200 & 52 & 15 & -28 \\
\hline $\mathrm{BF}_{2} \mathrm{dbm}(\mathrm{I}) \mathrm{PLA}$ & 405 & 450 & 535 & 0.54 & 4.37 & 71 & 24 & -24 \\
\hline
\end{tabular}

Table 1. Nanoparticle characteristics. ${ }^{a}$ Absorption maxima. ${ }^{b}$ Fluorescence emission maxima excited at $369^{\wedge} \mathrm{nm}$. 'Phosphorescence maxima in the delayed emission spectrum excited at $369 \mathrm{~nm}$ with a $0.5 \mathrm{~ms}$

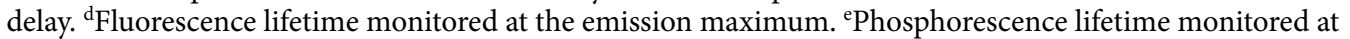
the phosphorescence maximum. f Hydrodynamic radius and polydispersity (PD) determined by dynamic light scattering. ${ }^{\text {ZZeta }}$ potential measured by ZetaSizer.

a

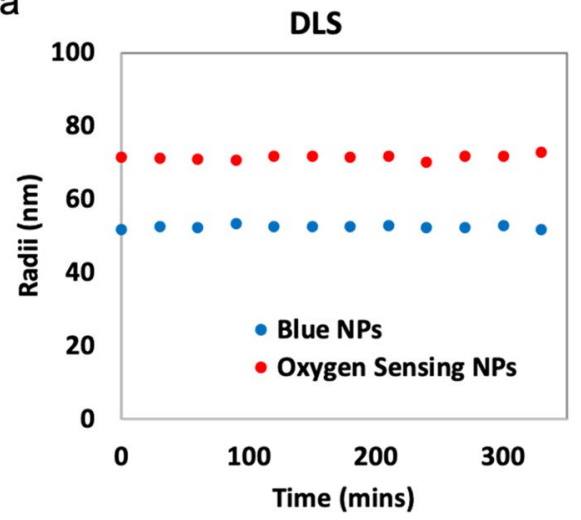

b

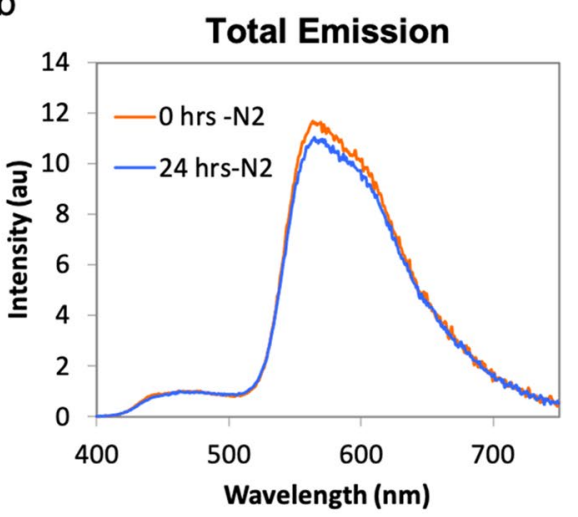

Figure 2. The stability of NPs. (a) DLS traces for NPs. The radii were recorded every $30 \mathrm{~min}$. (b) The stable oxygen-sensing ability of NPs was confirmed by measuring the emission before and after incubation at $37^{\circ} \mathrm{C}$ for $24 \mathrm{~h}$. 


\section{Blue NPs}

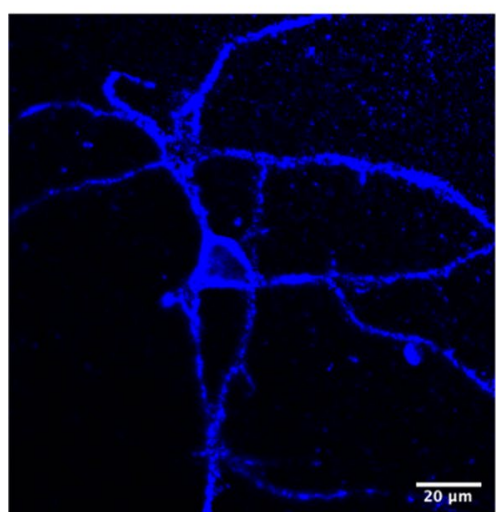

MitoTracker Red FM

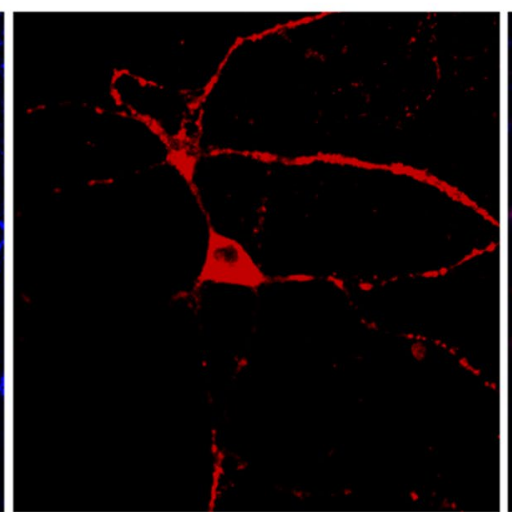

Merged Channel

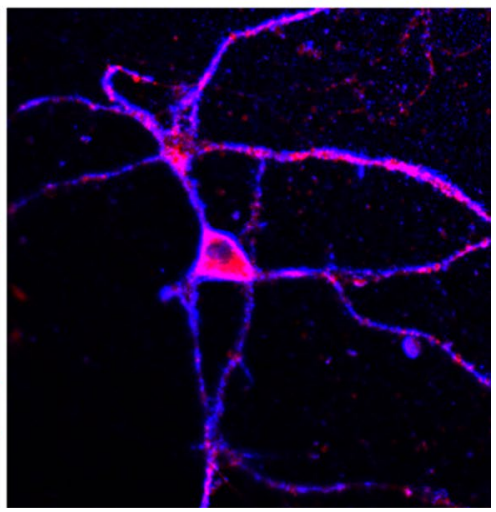

Figure 3. Neuronal imaging under confocal microscopy. The images showed labelling from blue NPs (left) and MitoTracker Red (middle) and merged channel from blue and red (right). The excitation wavelength is 405 and $595 \mathrm{~nm}$.

the methods) at a final concentration of $500 \mu \mathrm{g} / \mathrm{mL}$, monitored by DLS every $30 \mathrm{~min}$ over $6 \mathrm{~h}$, a period of time sufficient for incubation and imaging. It was observed that the NPs could maintain their size without aggregation for the duration (Fig. 2a). We also evaluated the optical stability of $\mathrm{O}_{2}$-sensing NPs. The emission of $\mathrm{O}_{2} \mathrm{NPs}^{\circ}$ suspended in ACSF solution was measured before and after incubation at $37^{\circ} \mathrm{C}$ for $24 \mathrm{~h}$. The two spectra were similar, indicating that these particles' phosphorescence $\mathrm{O}_{2}$-sensing ability was unaffected over the incubation period (Fig. 2b).

The blue NPs suspended in the neuronal medium were then used to label primary neuronal cultures due to their high quantum yield $\left(\Phi_{\mathrm{F}}=0.99\right)$, ideal for tracking cellular internalization ${ }^{27,28}$. The cultures were 14 DIV at the time of the experiment, with well-formed neuronal structures, including synapses. The mitochondria were labeled with MitoTracker Red FM, the maxima fluorescence of which $\left(\lambda_{\mathrm{F}}=644 \mathrm{~nm}\right)$ is far away from the blue NP emission. With confocal microscopy, we found that blue NPs showed non-specific and uniform interactions and distribution with living cells (e.g., neurons and glial cells). Neuronal cell bodies (i.e., a perinuclear region of cytoplasm), dendrites, and synapses can be easily visualized with the bright blue fluorescence (Fig. 3; left). To further ensure that the NPs enter the neurons and can measure intracellular oxygen level, a red mitochondria dye, MitoTracker Red FM, with emission having no interference with NPs fluorescence, was selected to label neurons (Fig. 3; middle). By specifically binding to the mitochondrial membrane, the MitoTracker targets mitochondria where the oxygen is consumed to generate ATP and energy for a variety of neuronal activities. As shown in Fig. 3, the blue channel of NPs emission and red channel of MitoTracker Red FM emissions were matched, revealing the colocalization of these two dyes. The colocalization of these two dyes suggested that effective intracellular oxygenation can be detected from the oxygen-sensing NPs.

Slice imaging and toxicity. Dual-emissive $\mathrm{O}_{2}$ sensing NPs possess oxygen-insensitive fluorescence and room temperature oxygen-sensitive phosphorescence. As oxygen concentration increases, the phosphorescence intensity decreases, whereas the fluorescence remains unchanging (Figure S1), serving as useful agents for ratiometric oxygen sensing ${ }^{29,32,33}$. At a range of $0-21 \% \mathrm{O}_{2}$, the $\mathrm{O}_{2}$ NPs had a linear relationship between the fluorescence to phosphorescence (F/P) ratio and oxygen concentration. After the cellular uptake studies in cultured neurons, $\mathrm{O}_{2}$ sensing NPs were tested using living brain slices. The brain slices were incubated with a solution containing $500 \mu \mathrm{g} / \mathrm{mL} \mathrm{O}_{2} \mathrm{NPs}$, during which they were oxygenated with $95 \% \mathrm{O}_{2}$. The dentate gyrus within the hippocampus was imaged because the neuronal circuits activated during seizures involve the hippocampus, in particular, the dentate granule cells (DGC), which regulate the flow of signal within the neuronal network. It is thus, of interest and importance to investigate how the oxygen level varies in the DGC layer. During imaging, the brain slice was sealed in a closed chamber with a circulation solution containing $95 \% \mathrm{O}_{2}$. The results were based on the analysis of a group of 10 slices from seven mice. As shown in Fig. 4a, the DGC layer is visible in brightfield, and the emission captured from fluorescence was indicative of the slice stained by nanoparticles. It was observed that the DGC layer has brighter fluorescence. This may be due to the great number of cells tightly packed here, resulting in more nanoparticles loaded. The phosphorescence was detected simultaneously along with fluorescence using a separate emission filter range (Fig. 4a).

The fluorescence and phosphorescence were analyzed to generate ratiometric images with an F/P ratio correlating with oxygen levels (Fig. $4 \mathrm{~b}$ and Figure S2). By taking the ratio of F/P, the uneven distribution of nanoparticles within the slice can be self-calibrated by the oxygen-insensitive fluorescence (i.e., internal standard). While the brain slice is supplied with oxygen, a subtle contrast between dendrites and cell bodies was observed; the oxygen level in dendrites is lower than in cell bodies of granule layers. The oxygen supply was stopped to test the NP response to oxygen changes in tissue, and imaging was taken every $5 \mathrm{~min}$ for $10 \mathrm{~min}$ (i.e., data points at 0 , $5,10 \mathrm{~min}$ ). The F/P ratio generally decreased over time (i.e., oxygen levels decreased), and the contrast between dendrites and cell bodies became more pronounced. Oxygen is consumed by mitochondria in neuronal cell bodies, at dendrites, and at synapses. At dendrites where synapses are located, there are many neuronal activities 
Brightfield
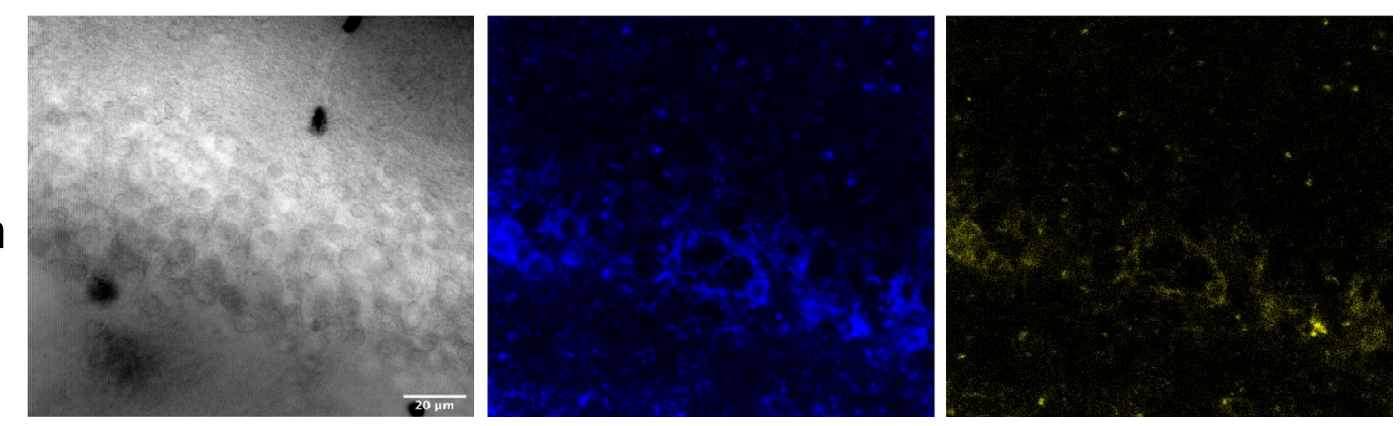

High $\left[\mathrm{O}_{2}\right]$
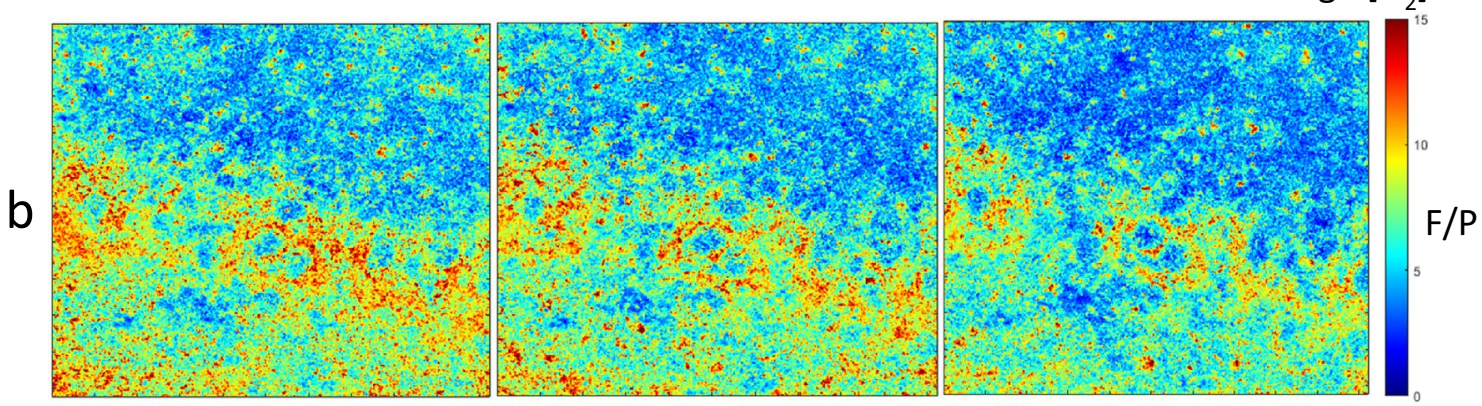

$\operatorname{Low}\left[\mathrm{O}_{2}\right]$

Figure 4. Brain slice imaging with $\mathrm{O}_{2}$ NPs. (a) Brightfield, fluorescence, and phosphorescence emission of the hippocampus DGC layer. (b) Ratiometric imaging when the oxygen supply is off over time.

\begin{tabular}{|l|l|l|l|l|l|l|l|l|l|l|l|l|l|}
\hline & Slice 2 & Slice 3 & Slice 4 & Slice 5 & Slice 6 & Slice 7 & Slice 8 & Slice 9 & Slice 10 & Slice 11 & Slice 12 & Median & SD \\
\hline $0 \mathrm{~min}$ & 1.0 & 1.0 & 1.0 & 1.0 & 1.0 & 1.0 & 1.0 & 1.0 & 1.0 & 1.0 & 1.0 & 1.0 & 0 \\
\hline $5 \mathrm{~min}$ & 1.448 & 1.207 & 1.033 & 1.742 & 0.903 & 1.443 & 1.625 & 1.449 & 1.882 & 1.098 & 0.903 & 1.443 & 0.335 \\
\hline $10 \mathrm{~min}$ & 2.029 & 1.685 & 1.064 & 2.304 & 0.887 & 1.084 & 1.478 & 1.478 & 2.470 & 1.241 & 1.115 & 1.478 & 0.533 \\
\hline
\end{tabular}

Table 2. Baseline ( $0 \mathrm{~min}$ )-normalized $\mathrm{F} / \mathrm{P}$ values per unit area below a threshold nanoparticle characteristics. The threshold was kept constant for all the slices. Null hypothesis H0: No observable difference of baseline normalized F/P per unit area between a pair of time instants. The normalized F/P at $0 \mathrm{~min}$ is non-normal, whereas KS test fails to reject that the normalized $\mathrm{F} / \mathrm{P}$ values are sampled from a Normal distribution in cases of $5 \mathrm{~min}(P=0.86)$ and $10 \mathrm{~min}(P=0.83)$. $\mathrm{H} 0$ is rejected when normalized $\mathrm{F} / \mathrm{P}$ values are compared at $0 \mathrm{~min}$ and $5 \mathrm{~min}(P=0.0077$, Wilcoxon rank-sum test) and at $(0 \mathrm{~min}, 10 \mathrm{~min})$ with $P=0.0005$ (Wilcoxon rank-sum test). Pairwise T-test fails to reject $\mathrm{H} 0$ for (5 min, $10 \mathrm{~min})$.

taking place; therefore, mitochondria are more concentrated there compared to cell bodies. The mitochondria rich synapses consume more oxygen and consume it more quickly, leading to lower oxygen levels.

The F/P ratio was determined in a region of interest encompassing dentate granule cells and their processes. The F/P ratios in 12 slices at $5 \mathrm{~min}$ and $10 \mathrm{~min}$ after oxygen supply to the slices was stopped shows increased phosphorescence at $5 \mathrm{~min}$ and $10 \mathrm{~min}$ with respect to the $\mathrm{F} / \mathrm{P}$ ratio at baseline (Table 2).

To further support the statement that oxygen is consumed at the site of synaptic activity, the potassium concentration in perfusion solution was increased from 2 to $5 \mathrm{mM}$. Potassium regulates neuronal activity, and an elevated extracellular $\mathrm{K}^{+}$level is known to affect synaptic potentials and increase neuronal activities ${ }^{34,35}$. We performed slice oxygen imaging under different potassium concentrations. When potassium concentration was increased, the regions away from the molecular layer were more hypoxic as suggested by the F/P ratio in the ratiometric oxygen imaging in Fig. 5a,b and Table 3.

The results from brain slice imaging demonstrated the effectiveness of oxygen sensing nanoparticles in studying brain activity by measuring intracellular oxygen levels.

These proof of principle studies performed using cultured neurons or acutely isolated brain slices revealed that the NPs could be effectively used to obtain insights into tissue oxygen levels. The efficacy of NPs in vivo remains to be determined. Evaluation of changes in tissue oxygenation in deeper brain structures may also be 
Brightfield

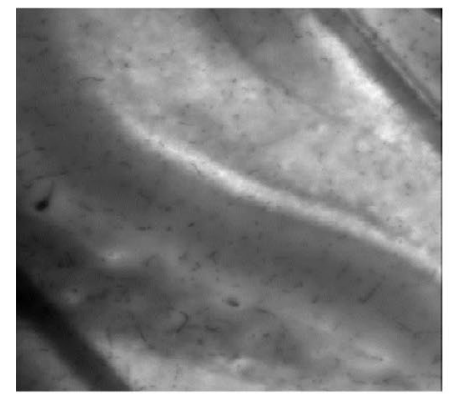

$2 \mathrm{mM}$

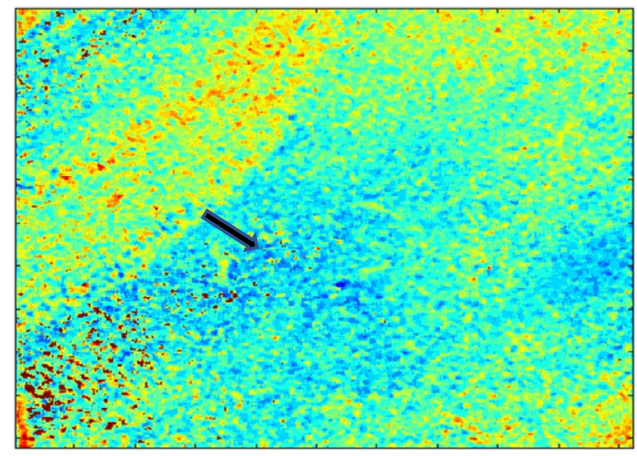

Fluorescence

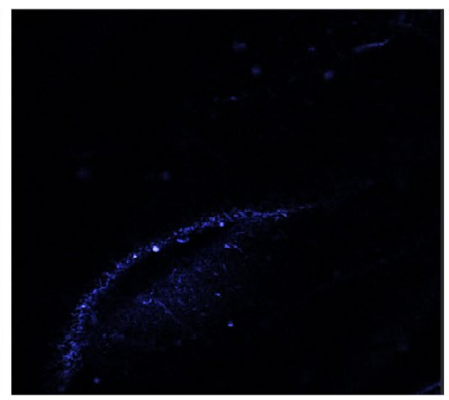

Phosphorescence

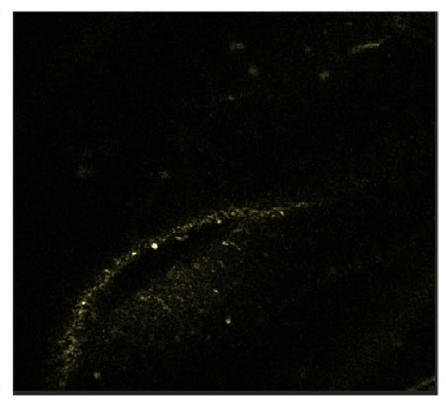

$5 \mathrm{mM}$

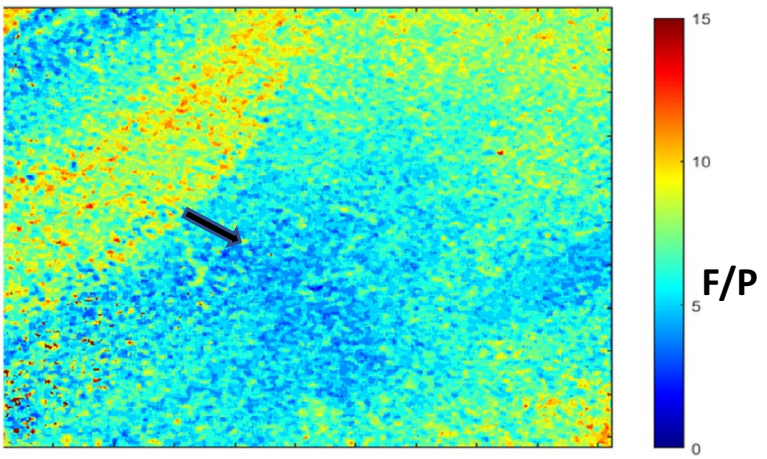

Low $\left[\mathrm{O}_{2}\right]$

Figure 5. Potassium concentration effect on neuronal activity. (a) Ratiometric images under low $\mathrm{K}^{+}(2 \mathrm{mM})$ and high $\mathrm{K}^{+}(5 \mathrm{mM})$ concentration. (b) Ratiometric imaging in the presence of $2 \mathrm{mM}$ and $5 \mathrm{mM}$ extracellular $\mathrm{KCl}$. Black arrows mark the regions with lower oxygen.

\begin{tabular}{|l|l|l|l|l|l|l|l|l|}
\hline & Slice 1 & Slice 2 & Slice 3 & Slice 4 & Slice 5 & Slice 6 & Median & SD \\
\hline $2 \mathrm{mM}$ & 1.0 & 1.0 & 1.0 & 1.0 & 1.0 & 1.0 & 1.0 & 0 \\
\hline $5 \mathrm{mM}$ & 2.029 & 1.121 & 1.080 & 1.203 & 1.107 & 1.035 & 1.114 & 0.3796 \\
\hline
\end{tabular}

Table 3. F/P per unit area below a threshold normalized to the $2 \mathrm{mM}$ measurement. Null hypothesis $\mathrm{H} 0$ : No observable difference of baseline normalized F/P per unit area between slices maintained in a solution containing $2 \mathrm{mM}$ and a $5 \mathrm{mM} \mathrm{K}$. The normalized $\mathrm{F} / \mathrm{P}$ at $0 \mathrm{~min}$ is non-normal, whereas $\mathrm{KS}$ test fails to reject that the normalized $\mathrm{F} / \mathrm{P}$ values are sampled from a Normal distribution in cases of $2 \mathrm{mM}$ solution $(P=0.23)$. Wilcoxon rank-sum test rejects $\mathrm{H} 0(P=0.022)$.

challenging and the effect of scattering and absorbance by of the fluorescence and phosphorescence signal will have to be resolved, likely through further modifications of the NPs.

For biomedical imaging, it is essential to verify that the imaging agent has no toxic effect on biological systems. The neurons in the DGC layer were recorded by a whole-cell patch-clamp to investigate the electrophysiological properties of the brain slice after the application of oxygen sensing nanoparticles. The data were collected in a group of five slices from four mice. As shown in Fig. 6 and Figure S3, the nanoparticle treated slice showed a nearly identical I-clamp trace with the control. Six membrane properties, including resting membrane potential, membrane resistance, membrane time constant, action potential wide, action potential threshold, action potential amplitude, were collected, all of which showed no significant difference between the nanoparticle treated slice and the control slice. The electrophysiology experiments confirmed that oxygen sensing nanoparticles did not measurably alter the electrophysiological properties of the neurons.

\section{Conclusions}

Oxygen nanosensors based on phosphorescence quenching can provide information about the brain with improved micron-level spatial and sub-seconds temporal resolution. In this work, the boron nanoparticles were used to study brain activity. Specifically, primary neuronal cell cultures were labeled by blue nanoparticles from $\mathrm{BF}_{2} \mathrm{dbmPLA}$ to confirm intracellular uptake. Electrophysiology experiments revealed that the application of nanoparticles did not have a significant effect on the electrophysiological properties of neurons. Oxygen sensing 
a

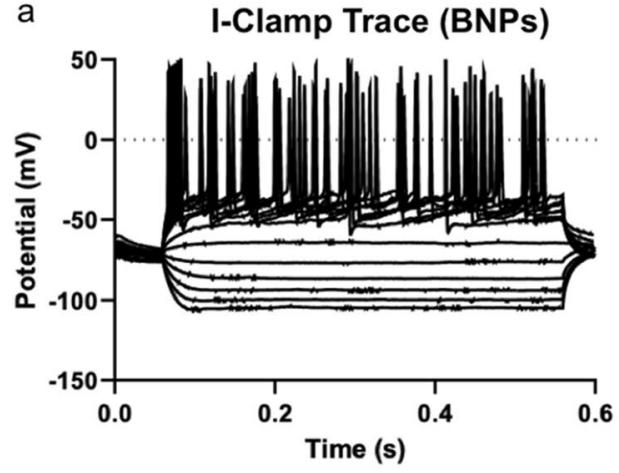

b

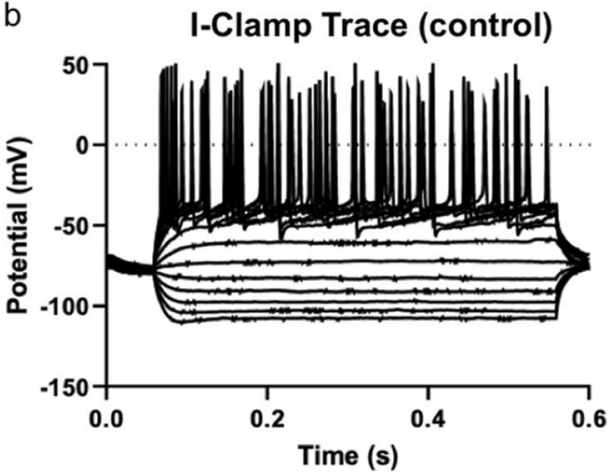

Figure 6. I-Clamp trace of neurons in the oxygen sensing nanoparticle treated slice (a) and the control slice (b).

nanoparticles based on $\mathrm{BF}_{2} \mathrm{dbm}(\mathrm{I}) \mathrm{PLA}$ was able to generate ratiometric oxygen maps for brain slices. In ex vivo brain slices, it was found that the neuronal cell bodies consume less oxygen than dendrites and synapses where mitochondria are more concentrated for synaptic activities. These data suggested the capability of boron nanoparticles serving as powerful and non-invasive sensing agents in the brain. Future work will involve oxygen imaging in the living animal and in combination with seizures induced via a cobalt model to study how oxygen levels are related to seizures.

\section{Methods}

All experiments involving mice were performed according to a protocol approved by the University of Virginia Animal Care and Use Committee (ACUC, protocol number 4093). All methods were carried out in accordance with relevant guidelines and regulations. The study was carried out in compliance with the ARRIVE guidelines. All chemicals were obtained from Sigma Aldrich (St. Louis, MO, USA) without further purification unless otherwise stated.

Dye-polymer synthesis and characterization. The boron dye-polymer was prepared according to a previously reported method ${ }^{26} .{ }^{1} \mathrm{H}$ NMR spectra were recorded on a Varian VMRS/600 $(600 \mathrm{MHz})$ instrument in $\mathrm{CDCl}_{3}$ unless otherwise indicated. ${ }^{1} \mathrm{H}$ NMR resonances were referenced to the residual protiochloroform signal at $7.260 \mathrm{ppm}$. Coupling constants are given in hertz. Polymer molecular weights (MW) and polydispersity indices (Đ) were determined by gel permeation chromatography (GPC) (THF, $25^{\circ} \mathrm{C}, 1.0 \mathrm{~mL} / \mathrm{min}$ ) using multiangle laser light scattering (MALLS) $\left(\lambda=658 \mathrm{~nm}, 25^{\circ} \mathrm{C}\right)$ and refractive index $(\mathrm{RI})\left(\lambda=658 \mathrm{~nm}, 25^{\circ} \mathrm{C}\right)$ detection. Polymer Laboratories $5 \mu \mathrm{m}$ mixed-C columns (guard column plus two columns) along with Wyatt Technology (Optilab T-rEX interferometric refractometer, miniDAWN TREOS multi-angle static light scattering (MALS) detector, ASTRA 6.0 software) and Agilent Technologies instrumentation (series 1260 HPLC with diode array (DAD) detector, ChemStation) were used in GPC analysis. The incremental refractive index $(d n / d c)$ was calculated by a single-injection method assuming $100 \%$ mass recovery from the columns. UV-vis spectra were recorded on a Hewlett-Packard 8452A diode-array spectrophotometer.

Luminescence measurements. Steady-state fluorescence spectra for the nanoparticle suspensions were recorded on a Horiba Fluorolog-3 Model FL3-22 spectrofluorometer (double-grating excitation and doublegrating emission monochromator) after excitation. Optically dilute aqueous solutions of the nanoparticles, with absorbance $<0.1 \mathrm{au}$, were prepared in $1 \mathrm{~cm}$ path length quartz cuvettes. Fluorescence spectra were obtained under ambient conditions (i.e., air, $\sim 21 \%$ oxygen in volume). For phosphorescence measurements, vials were capped with a $12 \mathrm{~mm}$ PTFE/silicone/PTFE seal (Chromatography Research Supplies) and were continuously purged with analytical grade $\mathrm{N}_{2}$ (Praxair) for 5 min before measurements. For $21 \% \mathrm{O}_{2}$ (i.e. air), measurements were taken under ambient conditions (open vial, no cap). Oxygen calibration was performed as previously described with Cole-Palmer flow gauges with mixtures of analytical grade $\mathrm{O}_{2}, \mathrm{~N}_{2}$, and $1 \% \mathrm{O}_{2}$ (in $\mathrm{N}_{2}$ ) gases $(\text { Praxair })^{29}$.

Nanoparticle fabrication. Nanoparticles were fabricated as previously reported ${ }^{36}$. The polymer $(\sim 3.0 \mathrm{mg})$ was dissolved in DMF $(3 \mathrm{~mL})$ and was added dropwise to rapidly stirred DI water $(27 \mathrm{~mL})$. The homogeneous mixture was stirred for $30 \mathrm{~min}$, and the nanoparticle suspensions were transferred into dialysis tubing (Specra/ Pro, 12-14 kDa MWCO, Fisher Scientific) followed by dialysis against DI water for $24 \mathrm{~h}$. Nanoparticle size and polydispersity were analyzed by dynamic light scattering (DLS, Wyatt, DynaPro). Zeta potentials were determined by Zetasizer Nano Z (Malvern Instruments, UK), and data were analyzed using DTS Nano software.

Nanoparticle stability. Nanoparticle stability was performed as previously described ${ }^{27}$. The stock suspensions of nanoparticles $(1 \mathrm{mg} / \mathrm{mL})$ were diluted $(500 \mu \mathrm{g} / \mathrm{mL})$ with regular neuronal medium (for cell labeling) and glucose (for slice labeling), respectively. Each sample $(100 \mu \mathrm{L})$ was injected into a 96 -well microtiter plate. Mineral oil was added on the top of each well via syringe to form a thin layer to prevent evaporation. The plate 
was put into the DLS instrument, protected from light, set to $37^{\circ} \mathrm{C}$, and the sizes and polydispersities of the nanoparticles were recorded over the course of $6 \mathrm{~h}$. For optical stability of $\mathrm{O}_{2} \mathrm{NPs}$, the total emission was measured before and after incubation.

Primary neuronal culture. Animals were handled according to a protocol approved by the University of Virginia Animal Care and Use Committee (ACUC), and efforts were made to minimize animal stress and discomfort. Cultures were prepared from postnatal day 0 to postnatal day 1 (P0-P1) C57BL/65 mice using methods described previously ${ }^{37}$. The newborn pups were decapitated, and their brains removed and placed in cold HEPES-buffered Hanks' balanced salt solution (HEPES-HBSS). The hippocampi were removed under a dissecting microscope and collected in a small petri dish containing HEPES-HBSS. The hippocampi were transferred to $0.125 \%$ trypsin HEPES-HBSS and were incubated for $15 \mathrm{~min}$ at $37^{\circ} \mathrm{C}$. Trypsin solution was centrifuged for $7 \mathrm{~min}$ at $700 \mathrm{rpm}$, and the supernatant was replaced with $5 \mathrm{~mL}$ HEPES-HBSS. The cells were rinsed with a warm surgical medium by centrifuging for $7 \mathrm{~min}$ at $700 \mathrm{rpm}$ and discarding the supernatant. Hippocampi were triturated until no fragments of tissue remained. Cell density was determined by trypan blue exclusion. Culture dishes were coated with poly-lysine and filled with $2 \mathrm{~mL}$ of surgical medium, which was prepared from Dulbecco's modified Eagles medium (DMEM) and F-12 supplement (1:1) (Invitrogen) with 10\% fetal bovine serum (heat-inactivated, Invitrogen), $2 \mathrm{mM} \mathrm{L}$-glutamine (Invitrogen), and penicillin-streptomycin (100 U/mL). Cells were plated at a density of 50,000 per $35 \mathrm{~mm}^{2}$ dishes and kept in a $5 \% \mathrm{CO}_{2}$ incubator at $37{ }^{\circ} \mathrm{C}$. After $24 \mathrm{~h}$, the surgical medium was changed to a serum-free neuronal regular medium containing DMEM and neurobasal (2.28:1) with $2 \%$ B27 and $2 \mathrm{mM}$ glutamine. The medium was replaced with fresh regular medium every two days.

Cell labeling. Primary neurons were 14 days in vitro (DIV) at the time of imaging for best observation of synapses. Blue NPs were diluted in a regular medium to yield a final concentration of $500 \mu \mathrm{g} / \mathrm{mL}$. Cells were incubated in the above solution at $37^{\circ} \mathrm{C}$ in a $5 \% \mathrm{CO}_{2}$ incubator for $1 \mathrm{~h}$. After staining, the cells were washed twice with HEPES-ACSF (pH 7.4, osmolarity 314-316 mOsm) containing (in mM) $147 \mathrm{NaCl}, 2.6 \mathrm{KCl}, 2 \mathrm{CaCl}_{2}$, $3 \mathrm{MgCl}_{2}, 10$ glucose, $10 \mathrm{HEPES}$ and were kept in such medium during imaging. To label mitochondria, cells were incubated with a regular medium containing MitoTracker Red FM (250 nM) for an additional 30 min before washing with HEPES-ACSF.

Brain slice preparation. Acutely isolated brain slices were prepared as previously described ${ }^{38}$. The animals were sacrificed under anesthesia, and the brains were removed and immersed immediately in ice-cold $\left(4{ }^{\circ} \mathrm{C}\right)$ oxygenated $\left(95 \% \mathrm{O}_{2}, 5 \% \mathrm{CO}_{2}\right.$ ) sucrose-ACSF ( $\mathrm{pH} 7.4$, osmolarity 300-310 mOsm) containing (in $\mathrm{mM}$ ) 56.5 $\mathrm{NaCl}, 2 \mathrm{KCl}, 5 \mathrm{MgSO}_{4}, 25 \mathrm{NaHCO}_{3}, 1 \mathrm{KH}_{2} \mathrm{PO}_{4}, 0.5 \mathrm{CaCl}_{2}, 10$ glucose, 113 sucrose. Coronal hippocampal slices $(200 \mu \mathrm{m})$ were prepared with a vibratome (VT1200S; Leica, Wetzlar, Germany) and were incubated in oxygenated sucrose-ACSF at $34^{\circ} \mathrm{C}$ for $30 \mathrm{~min}$ before labeling or recording.

Slice labeling. To minimize NP aggregation, $\mathrm{O}_{2}$ sensing NPs were suspended in $3 \mathrm{M}$ glucose with NP final concentration at $500 \mu \mathrm{g} / \mathrm{mL}$. Slices were labeled by incubating in the oxygenated NP glucose solution for $2 \mathrm{~h}$. Slices were washed and kept in oxygenated sucrose-ACSF prior to imaging.

Imaging. Imaging was performed using a Zeiss 780 confocal/multiphoton microscope system at the UVA Keck Imaging Center with Zeiss Zen software for image acquisition (Carl Zeiss, Oberkochen, Germany). For imaging cultured neurons, the excitation wavelengths used to visualize blue NPs and MitoTracker Red FM were $405 \mathrm{~nm}$ and $595 \mathrm{~nm}$, respectively. Emission filters ranged from 410 to $500 \mathrm{~nm}$ for blue, and $600 \mathrm{~nm}$ to $700 \mathrm{~nm}$ for red. Magnifications of $40 \times$ or higher were used in regions of interest. For imaging live slices, the brain slices were anchored in a closed bath chamber and perfused with oxygenated sucrose-ACSF. A $405 \mathrm{~nm}$ laser was used as the excitation source, and emission ranged from 410 to $500 \mathrm{~nm}$ to capture fluorescence and $500 \mathrm{~nm}$ to $650 \mathrm{~nm}$ to capture phosphorescence. For high $\mathrm{K}^{+}$measurements, the $\mathrm{K}^{+}$concentration was increased from 2 to $5 \mathrm{mM}$ in the perfusion solution ( $56.5 \mathrm{NaCl}, 5 \mathrm{KCl}, 5 \mathrm{MgSO}_{4}, 25 \mathrm{NaHCO}_{3}, 1 \mathrm{KH}_{2} \mathrm{PO}_{4}, 0.5 \mathrm{CaCl}_{2}, 10$ glucose, 107 sucrose). Ratiometric imaging was processed in MATLAB by taking the ratio of fluorescence to phosphorescence.

Electrophysiology. Electrophysiological properties of DGC neurons were recorded using a whole-cell patch-clamp technique as described previously ${ }^{39}$. Animals were aged around four weeks at the time of the study. The slices were perfused with oxygenated ACSF. Current-clamp recordings were performed for the measurement of cell membrane properties, and for filling biocytin into the cell. Recordings were performed in tight-seal (seal $\geq 1$ Gigohm) current-clamp mode. The recording solution ACSF (in mM): $126 \mathrm{NaCl}, 2.5 \mathrm{KCl}, 26 \mathrm{NaHCO}_{3}$, $2 \mathrm{CaCl}_{2}, 2 \mathrm{MgCl}_{2}, 1.25 \mathrm{NaH}_{2} \mathrm{PO}_{4}, 10$ Glucose. The internal solution was (in mM): $135 \mathrm{~K}$-gluconate, $7 \mathrm{KCl}, 10$ HEPES, 0.5 EGTA, 2.5 NaCl, $4 \mathrm{Mg}$-ATP, and 0.3 Na-GTP. Electrode capacitance was electronically compensated. Access resistance was continuously monitored, and if the series resistance increased by $20 \%$ at any time, the recording was terminated. Currents were filtered at $2 \mathrm{kHz}$, digitized using a Digidata 1322 digitizer (Molecular Devices, Sunnyvale, CA, USA), and acquired using Clampex 10.2 software (Molecular Devices). All recordings were performed at $30^{\circ} \mathrm{C}$. The currents were analyzed as described before using the MiniAnalysis software.

\section{Data availability}

The datasets generated and analyzed in this work are available from the corresponding author upon reasonable request. 
Received: 28 August 2020; Accepted: 17 December 2020

Published online: 13 January 2021

\section{References}

1. Weiss, H. M. Appreciating oxygen. J. Chem. Educ. 85, 1218-1219 (2008).

2. Semenza, G. L. Life with oxygen. Science (80-) 318, 62-64 (2007).

3. Rich, P. R. The molecular machinery of Keilin's respiratory chain. Biochem. Soc. Trans. 31, 1095-1105 (2003).

4. Mink, J. W., Blumenschine, R. J. \& Adams, D. B. Ratio of central nervous system to body metabolism in vertebrates: its constancy and functional basis. Am. J. Physiol. Regul. Integr. Comp. Physiol. 10, 203-212 (1981).

5. Harris, J. J., Jolivet, R. \& Attwell, D. Synaptic energy use and supply. Neuron 75, 762-777 (2012).

6. Devine, M. J. \& Kittler, J. T. Mitochondria at the neuronal presynapse in health and disease. Nat. Rev. Neurosci. 19, 63-80 (2018).

7. Neoptolemos, J. P. et al. Whole-body hypothermia for neonates with hypoxic-ischemic encephalopathy seetha. N. Engl. J. Med. 350, 1200-1210 (2004).

8. Tang, Y., Lu, A., Aronow, B. J. \& Sharp, F. R. Blood genomic responses differ after stroke, seizures, hypoglycemia, and hypoxia: blood genomic fingerprints of disease. Ann. Neurol. 50, 699-707 (2001).

9. Prass, K. et al. Hypoxia-induced stroke tolerance in the mouse is mediated by erythropoietin. Stroke 34, 1981-1986 (2003).

10. Glass, H. C. et al. Clinical neonatal seizures are independently associated with outcome in infants at risk for hypoxic-ischemic brain injury. J. Pediatr. 155, 318-323 (2009).

11. Wirrell, E. C., Armstrong, E. A., Osman, L. D. \& Yager, J. Y. Prolonged seizures exacerbate perinatal hypoxic-ischemic brain damage. Pediatr. Res. 50, 445-454 (2001).

12. Baud, O. et al. Gestational hypoxia induces white matter damage in neonatal rats: a new model of periventricular leukomalacia. Brain Pathol. 14, 1-10 (2004).

13. Ogunshola, O. O. \& Antoniou, X. Contribution of hypoxia to Alzheimer's disease: Is HIF-1a a mediator of neurodegeneration?. Cell. Mol. Life Sci. 66, 3555-3563 (2009).

14. Zhang, X. \& Le, W. Pathological role of hypoxia in Alzheimer's disease. Exp. Neurol. 223, 299-303 (2010).

15. Roussakis, E., Li, Z., Nichols, A. J. \& Evans, C. L. Oxygen-sensing methods in biomedicine from the macroscale to the microscale. Angew. Chem. Int. Ed. 54, 8340-8362 (2015).

16. Li, Z. et al. Non-invasive transdermal two-dimensional mapping of cutaneous oxygenation with a rapid-drying liquid bandage. Biomed. Opt. Express 5, 3748-3764 (2014).

17. Nichols, A. J., Roussakis, E., Klein, O. J. \& Evans, C. L. Click-assembled, oxygen-sensing nanoconjugates for depth-resolved, nearinfrared imaging in a 3-D cancer model. Angew. Chem. Int. Ed. 53, 3671-3674 (2014).

18. Wang, X. D. \& Wolfbeis, O. S. Optical methods for sensing and imaging oxygen: materials, spectroscopies and applications. Chem. Soc. Rev. 43, 3666-3761 (2014)

19. Wolfbeis, O. S. Luminescent sensing and imaging of oxygen: fierce competition to the Clark electrode. BioEssays 37, 921-928 (2015).

20. Roussakis, E., Li, Z., Nowell, N. H., Nichols, A. J. \& Evans, C. L. Bright, 'clickable' porphyrins for the visualization of oxygenation under ambient light. Angew. Chem. Int. Ed. 54, 14728-14731 (2015).

21. Mauleon, G., Fall, C. P. \& Eddington, D. T. Precise spatial and temporal control of oxygen within in vitro brain slices via microfluidic gas channels. PLoS ONE 7, 43309 (2012).

22. Zhang, C., Bélanger, S., Pouliot, P. \& Lesage, F. Measurement of local partial pressure of oxygen in the brain tissue under normoxia and epilepsy with phosphorescence lifetime microscopy. PLoS ONE 10, 0135536 (2015).

23. Tsytsarev, V. et al. In vivo imaging of brain metabolism activity using a phosphorescent oxygen-sensitive probe. J. Neurosci. Methods 216, 146-151 (2013).

24. Ingram, J. M., Zhang, C., Xu, J. \& Schiff, S. J. FRET excited ratiometric oxygen sensing in living tissue. J. Neurosci. Methods 214, 45-51 (2013).

25. Dmitriev, R. I. et al. Imaging oxygen in neural cell and tissue models by means of anionic cell-permeable phosphorescent nanoparticles. Cell. Mol. Life Sci. 72, 367-381 (2015).

26. Zhang, G. et al. Multi-emissive difluoroborondibenzoylmethanepolylactide exhibiting intense fluorescence and oxygen-sensitive room-temperature phosphorescence. J. Am. Chem. Soc. 129, 8942-8943 (2007).

27. Belanger, M. C. et al. Labelling primary immune cells using bright blue fluorescent nanoparticles. Biomater. Sci. 8, 1897-1909 (2020).

28. Pfister, A., Zhang, G., Zareno, J., Horwitz, A. F. \& Fraser, C. L. Boron polylactide nanoparticles exhibiting fluorescence and phosphorescence in aqueous medium. ACS Nano 2, 1252-1258 (2008).

29. Zhang, G., Palmer, G. M., Dewhirst, M. W. \& Fraser, C. L. A dual-emissive-materials design concept enables tumour hypoxia imaging. Nat. Mater. 8, 747-751 (2009).

30. Liao, L. et al. A convergent synthetic platform for single-nanoparticle combination cancer therapy: ratiometric loading and controlled release of cisplatin, doxorubicin, and camptothecin. J. Am. Chem. Soc. 136, 5896-5899 (2014).

31. Dante, S. et al. Selective targeting of neurons with inorganic nanoparticles: revealing the crucial role of nanoparticle surface charge. ACS Nano 11, 6630-6640 (2017).

32. DeRosa, C. A. et al. Oxygen sensing difluoroboron $\beta$-diketonatepolylactide materials with tunable dynamic ranges for wound imaging. ACS Sens, 1, 1366-1373 (2016).

33. DeRosa, C. A. et al. Oxygen sensing difluoroborondinaphthoylmethanepolylactide. Macromolecules 48, 2967-2977 (2015).

34. Takahashi, S., Shibata, M. \& Fukuuchi, Y. Effects of increased extracellular potassium on influx of sodium ions in cultured rat astroglia and neurons. Dev. Brain Res. 104, 111-117 (1997).

35. Larsen, B. R., Stoica, A. \& MacAulay, N. Managing brain extracellular $\mathrm{K}^{+}$during neuronal activity: the physiological role of the $\mathrm{Na}^{+} / \mathrm{K}^{+}$-ATPase subunit isoforms. Front. Physiol. 7, 141 (2016).

36. Kerr, C. et al. Luminescent difluoroboron $\beta$-diketonate PLA-PEG nanoparticles. Biomacromol 18, 551-561 (2017).

37. Johnson, S. E., Hudson, J. L. \& Kapur, J. Synchronization of action potentials during low-magnesium-induced bursting. J. Neurophysiol. 113, 2461-2470 (2015)

38. Joshi, S. et al. A novel therapeutic approach for treatment of catamenial epilepsy. Neurobiol. Dis. 111, 127-137 (2018).

39. Sun, J. \& Kapur, J. M-type potassium channels modulate Schaffer collateral-CAl glutamatergic synaptic transmission. J. Physiol. 590, 3953-3964 (2012).

\section{Acknowledgements}

We would like to thank UVA Brain Institute for supporting this work. We have acquired images using the UVA Keck Center Zeiss 780 Confocal microscopy system (NIH OD016446; PI: AP). 


\section{Author contributions}

C.L.F. designed the nano particles. M.Z. synthesized and characterized the materials, performed the stability study, prepared the brain slices and conducted oxygen imaging experiments. J. K. designed the experiments to test the nanoparticles in brain slices and cultured neurons. M.Z. and S.J. prepared the neuronal cultures. T.B. processed the ratiometric imaging in MATLAB and performed the quantification. H.S. performed the electrophysiological experiment. M.Z and J. K. wrote the manuscript. All co-authors reviewed the manuscript.

\section{Competing interests}

The authors declare no competing interests.

\section{Additional information}

Supplementary Information The online version contains supplementary material available at https://doi. org/10.1038/s41598-020-80172-w.

Correspondence and requests for materials should be addressed to C.L.F. or J.K.

Reprints and permissions information is available at www.nature.com/reprints.

Publisher's note Springer Nature remains neutral with regard to jurisdictional claims in published maps and institutional affiliations.

(c) (i) Open Access This article is licensed under a Creative Commons Attribution 4.0 International License, which permits use, sharing, adaptation, distribution and reproduction in any medium or format, as long as you give appropriate credit to the original author(s) and the source, provide a link to the Creative Commons licence, and indicate if changes were made. The images or other third party material in this article are included in the article's Creative Commons licence, unless indicated otherwise in a credit line to the material. If material is not included in the article's Creative Commons licence and your intended use is not permitted by statutory regulation or exceeds the permitted use, you will need to obtain permission directly from the copyright holder. To view a copy of this licence, visit http://creativecommons.org/licenses/by/4.0/.

(c) The Author(s) 2021 